\title{
Processo iterativo de design paramétrico e análise estrutural aplicado ao desenvolvimento de estrutura de torre eólica
}

Iterative process of parametric design and structural project applied to the development of lattice wind tower

\author{
> Marina Ferreira Borges \\ DEES UFMG, Brasil \\ marinafborges@gmail.com
}

\author{
> Ricardo Hallal Fakury \\ DEES UFMG, Brasil \\ fakury@dees.ufmg.br
}

\begin{abstract}
This article proposes to study the process of parametric design integrated analysis and structural design. This application model is called Performative Model; the form is generated based on performance criteria. The digital tools facilitate the information flow between designers using parametric model and Finite Element Analysis. To research the method of Performative Model is proposed the development of a conceptual framework of lattice wind tower with the aim of a quantitative and qualitative structure optimization. Therefore, the parametric modeling will be done using Rhinoceros software, the plugin for creating algorithms Grasshoper and structural analysis plugin Scan \& Solve.
\end{abstract}

Keywords: Performative model; Parametric model; Finite Element Analysis; Lattice Wind Tower.

\section{Introdução}

O desenvolvimento de tecnologias digitais tem possibilitado mudanças significativas nos processos de projeto de arquitetura, engenharia e na indústria da construção. A tecnologia CAD (Computer Aided Design) permitiu o desenvolvimento de técnicas de representação 2D e 3D em ambiente digital e a tecnologia BIM (Building Information Modeling) significou o acréscimo de informaçóes aos modelos através de relações paramétricas. Até então as tecnologias CAD e BIM continuam associadas a um processo tradicional de projeto, onde existe uma sequência de decisôes que envolvem síntese da forma arquitetônica e posterior avaliaçáo, que no caso do projeto de estruturas, acontece através de análise estrutural. Numa abordagem contemporânea para o projeto estrutural, a sequência de decisóes parte da estrutura e do material, para posteriormente ser definida a forma. Esta abordagem foi denominada por Oxman (2010) como Novo Estruturalismo, onde o desenvolvimento de estruturas complexas só é possível com a reversão no modo de pensar o processo de geraçáo da forma. Desta maneira, a participaçáo do engenheiro estrutural deixa de ser somente na fase de avaliação e aparece nos primeiros estágios de geração da forma, criando um processo de pesquisa e produçáo do conhecimento comum entre arquitetos e engenheiros.

Neste trabalho será investigada a metodologia abordada por Kolarevic (2005) de Modelo Performativo, onde serão aplicadas ferramentas de design paramétrico e análise estrutural. Nessa abordagem, que tem como base de desenvolvimento o desempenho, as estratégias qualitativas e quantitativas são racionalizadas, e a relação entre material, estrutura e forma resulta em uma estética relacionada com economia e eficiência.

A literatura fornece exemplos de processos de Modelos Performativos tal como o desenvolvimento da cobertura de vidro do British Museum em Londres (Burry, 2010), concebida por Foster + Partners. Sua definição geométrica consistiu de duas partes: a primeira, na definição da forma da superfície; e a segunda, no desenvolvimento do padráo da estrutura metálica (Fig. 1). Para a concepção da forma, foi necessária a criação de uma fórmula matemática que garantisse a singularidade da curvatura no contorno, já que a cobertura seria executada em um edifício histórico existente. Uma vez encontrada a forma, foram aplicados algoritmos de otimização, o que permitiu manipular a malha original de controle e testar geometricamente diferentes opçóes do grid estrutural. A cada peça foi atribuída uma dimensão de seçáo ideal, o que foi posteriormente revisto dentro do processo automatizado computacional para o tamanho mais próximo dos perfis disponíveis comercialmente.

\section{Aplicação do Modelo Performativo}

Como objeto de estudo e experimentação para aplicação da metodologia de Modelo Performativo, propóe-se a concepção da estrutura de uma torre treliçada de energia eólica. Para o desenvolvimento da forma serão aplicados os principais parâmetros que classificam esses modelos: geometria, técnicas de modelagem geométrica, análise de tensóes por método dos elementos finitos, interoperabilidade e colaboração entre profissionais de arquitetura e engenharia. 


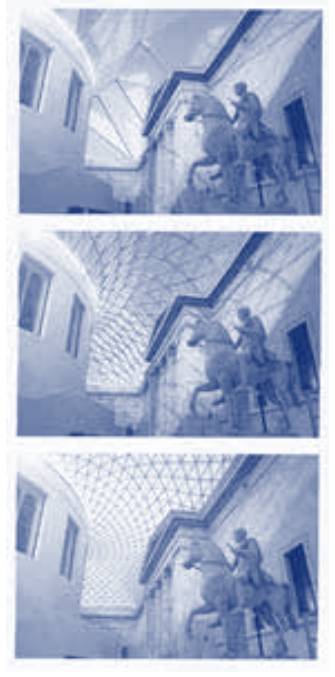

Figura l: Simulaçôes computacionais de subdivisão da cobertura. (Burry, 2010).

\section{Geometria}

Para a geração da geometria da torre foram estabelecidos os seguintes parâmetros:

Tabela 1: Parâmetros de dimensionamento da torre

\begin{tabular}{|l|l|}
\hline Altura total & 140 metros \\
Altura da base & 80 metros \\
Altura do topo & 60 metros \\
Diâmetro da base & variação de 25 a 35 metros \\
Diâmetro intermediário & 5 metros \\
Diâmetro do topo & variação de 2,5 a 5 metros \\
\hline
\end{tabular}

Optou-se por adotar o desenvolvimento da geometria em estrutura treliçada de aço com perfis laminados tubulares circulares (Vallourec Tubos do Brasil, 2014), devido à sua capacidade de responder a momento fletor em vários eixos, necessária em formas verticais onde forças laterais e cargas verticais excêntricas atuam simultaneamente. Além disto, a treliça espacial permite a variação geométrica ao longo de um eixo vertical possibilitando inúmeras variaçóes com relação ao design.

Para disposição da forma da torre e distribuição de barras, foi adotada a geometria do hiperboloide de uma folha. Esta geometria permite a manutenção da integridade estrutural com a redução do uso de materiais, pois em cada um de seus pontos há duas retas distintas que cruzam a superfície. A construção de outras torres configuradas em hiperboloide de uma folha também serviu como referência da pesquisa (Quadro 1). O projeto da Torre de Canton pode ser considerado um exemplo de Modelo Performativo, visto que a estrutura foi resultado de um processo colaborativo entre os arquitetos holandeses Mark Hemel e Barbara Kuit com a empresa de engenharia britânica Arup, que se responsabilizou pelo cálculo estrutural. A forma foi desenvolvida com o auxílio de ferramentas digitais de design paramétrico, de tal maneira a se buscar a otimização da estrutura.

Quadro 1: Torres construídas com hiperboloide de uma folha

\begin{tabular}{|c|c|c|}
\hline Torre Shabolovka & Torre de Kobe & Torre de Canton \\
\hline País: Rússia & País: Japão & País: China \\
\hline Construção: 1920 & Construção: 1963 & Construção: 2009 \\
\hline Altura: 160 metros & Altura: 108 metros & Altura: 488 metros \\
\hline
\end{tabular}

\section{Técnicas}

No estudo da torre de energia eólica, ter-se-ão duas etapas de geração e análise do modelo paramétrico. $\mathrm{Na} 1^{\text {a }}$ etapa, a estrutura foi modelada como casca com geometria de espessura constante, onde foram testados diversos modelos, variando os diâmetros de base e topo, e a sua espessura. Esta foi analisada elasticamente pelo MEF para obtenção da tensão de Von Mises.

$\mathrm{Na} 2^{\text {a }}$ etapa, após a definição da espessura, essa casca foi transformada em uma estrutura de barras treliçada equivalente. Para isto, foram aplicadas as definições de barras, moduladas nos sentidos vertical, horizontal e inclinadas. Através de programação algorítmica, a quantidade de barras foi o resultado da espessura da casca selecionada, e os diâmetros dos tubos foram automaticamente selecionados de acordo com os critérios adotados.

\section{la etapa}

Para gerar a geometria inicial da casca (Fig. 2), foram estabelecidos os parâmetros de dimensionamento exigidos para o projeto da estrutura da torre eólica (Tabela 1).

Para a modelagem geométrica foi adotado o plugin Grasshopper para Rhinoceros, onde foi feita a montagem do algoritmo para a geração da forma. Os dados iniciais foram a locaçáo das coordenadas dos pontos de acordo com as alturas fornecidas, e posteriormente os raios dos círculos gerados a partir desses pontos.

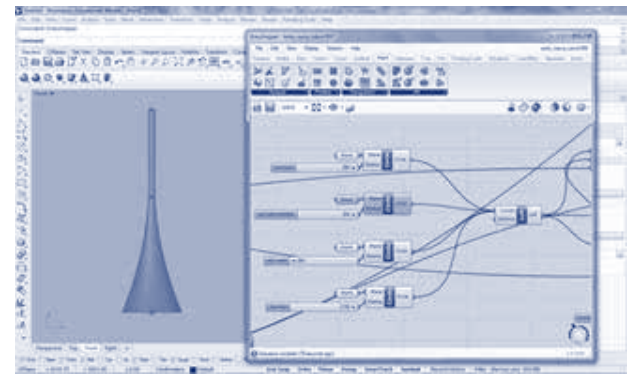

Figura 2: Algoritmo de desenvolvimento da casca (elaborado pelos autores) 
Para avaliaçáo da forma, tanto na $1^{\text {a }}$, quanto na $2^{\mathrm{a}}$ etapa, os modelos seráo analisados pelo MEF utilizando o plugin Scaner Solve. A vantagem de se utilizar os plug-ins Grasshoper e Scan\&solve é a interoperabilidade, tendo sua base de funcionamento no programa Rhinoceros, evitando que o sólido seja exportado para outro programa e que haja a necessidade de readaptação da malha.

Análise de tensões pelo Método dos

Elementos Finitos - la etapa

A análise de tensóes da casca foi feita aplicando-se os seguintes dados:

Quadro 3. Parâmetros para análise estrutural

\begin{tabular}{l|r|}
\hline aço & VMB-350 \\
\cline { 2 - 2 } $\begin{array}{l}\text { resistência ao escoamento } \\
\text { resistência à ruptura }\end{array}$ & $350 \mathrm{MPa}$ \\
\cline { 2 - 2 } carga do equipamento & $485 \mathrm{MPa}$ \\
\hline $\begin{array}{l}\text { vento no equipamento } \\
\text { momento total (binário) }\end{array}$ & $2,780 \times 10^{6} \mathrm{~N}$ \\
\hline
\end{tabular}

Após o teste com espessuras variando de 5 a $50 \mathrm{~cm}$, verificouse que o limite de espessura da casca é de $8 \mathrm{~cm}$, já que a casca com $7 \mathrm{~cm}$ excede o limite da tensão de Von Mises. Portanto, a análise de tensóes da casca com $8 \mathrm{~cm}$ foi considerada como o limite para o desenvolvimento da torre em estrutura treliçada (Fig. 3).

\section{$2^{\text {a }}$ etapa}

O objetivo da segunda etapa foi gerar a treliça com perfis tubulares circulares a partir da casca selecionada. Como primeiro parâmetro para geração da treliça, o domínio da superfície foi subdividido em módulos horizontais. À princípio, foram estabelecidos 7 módulos, tendo 20 metros cada.

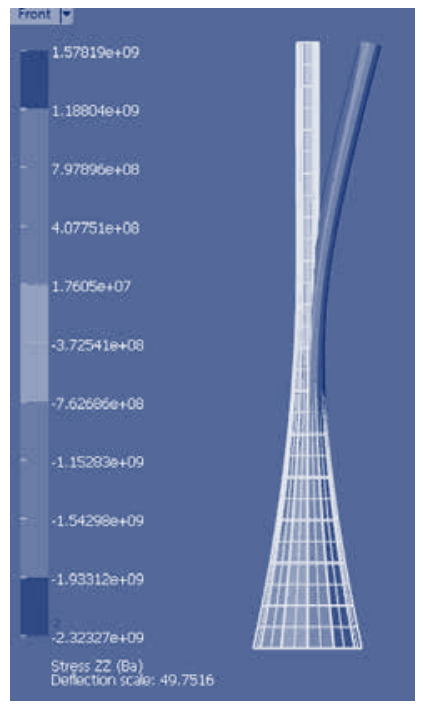

Figura 3: Análise de tensôes para casca com espessura de $8 \mathrm{~cm}$ (elaborado pelos autores)
A divisão dos módulos verticais foi feita pela programação de parâmetros através de inserção de programação orientada a objetos em código Visual Basic (VB), dentro do Grasshopper. Esse código possui parâmetros de entrada e condicionais de saída. Como parâmetros de entrada, foi definida que a área do anel gerado através da escolha da geometria da casca, seria equivalente à área do somatório da área dos tubos no diâmetro intermediário da superfície, resultando em um valor de $N$ tubos necessários..

O valor $N$ será responsável pela geração de pontos na superfície, distribuídos ao longo dos círculos gerados pela modulação horizontal. Por estar parametrizado, o valor $N$ pode ser facilmente alterado, redimensionando de maneira iterativa toda a treliça final. O código VB (Fig. 4) tem também como valor de entrada a tabela comercial de tubos circulares da VALLOUREC. Foram utilizados para as barras primárias (tubos de maior diâmetro na vertical ou ligeiramente inclinados) diâmetros entre 219,1 mm e 355,6 mm. Para as barras secundárias (diagonais necessárias para estabilizar adequadamente a estrutura), ficou estabelecido que fossem da ordem da metade do diâmetro comercial das barras primárias, portanto, variando entre 101,6 mm e 219,1 mm. As tabelas foram divididas em duas saídas, mas possuem através do código uma interdependência com relação às seus índices, para garantir assim que os perfis primários tenham seu valor respectivamente equivalente ao perfil secundário.

No código textual, foram declaradas as variáveis para resultar em uma geometria associativa com as condicionais estabelecidas. Dessa maneira, a espessura da casca fica totalmente vinculada aos tubos que serão gerados na treliça. A alteração da espessura da casca gera automaticamente a alteração da quantidade de tubos, sua distribuição ao longo da modulação horizontal, e dos diâmetros de perfis primários e secundários. Esse valor está diretamente associado à lista de diâmetros comerciais, tendo como resultado a forma associada aos perfis com os quais ela será produzida.

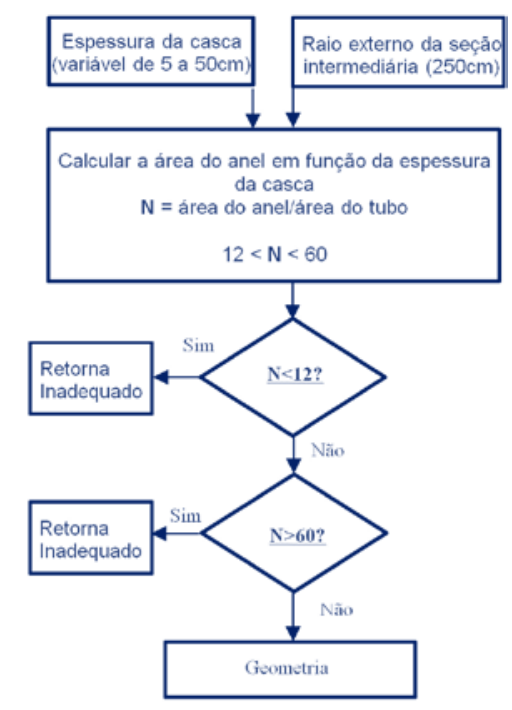

Diagrama l: Condicional para geração da treliça. 
Os módulos teráo os pontos de saída rotacionados em 30o com relação aos pontos da sequência da modulação inferior. Para tanto, os pontos de saída foram divididos em uma matriz, no formato "árvore", onde os pontos finais da primeira modulação da base coincidem com os pontos iniciais da segunda modulação, e assim consequentemente até o topo.

$\mathrm{Na}$ Figura 4 pode-se visualizar a montagem do algoritmo para a geometria à direita, e a esquerda, a treliça resultante relativa à geometria da casca.

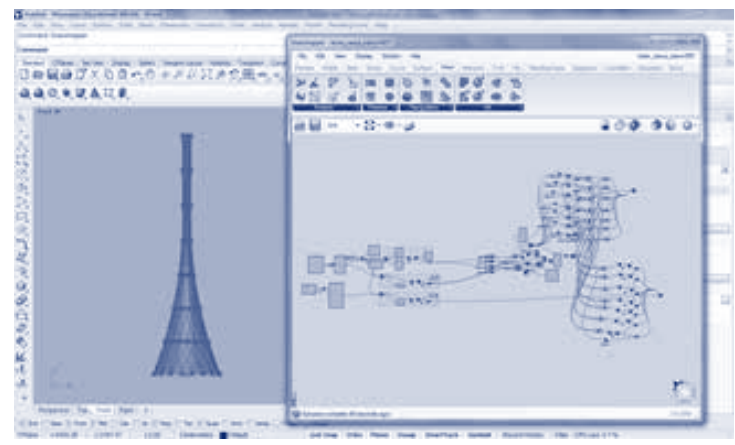

Figura 4: Algoritmo de desenvolvimento da treliça (elaborado pelos autores)

Mesmo após à geração da treliça, a espessura inicial da casca pode ser facilmente alterada, gerando novamente uma nova forma de treliça. Esta alteraçáo da forma pode ser feita quantas vezes forem necessárias, já que o modelo tridimensional não é resultado de uma modelagem geométrica, mas de uma modelagem através de algoritmo com dados parametrizáveis. Sendo assim, a análise estética e de tensóes da geometria pode ocorrer de maneira iterativa, visto que não demanda um retrabalho de modelagem.

Para exemplificar, o Quadro 2 mostra um teste com três possibilidades de treliça através da alteração de parâmetros:

Quadro 2: Teste de possibilidades da treliça através de alteração de parâmetros.

\begin{tabular}{|c|c|c|}
\hline Esp. casca: 25cm & Esp. casca: $35 \mathrm{~cm}$ & Esp. casca: $45 \mathrm{~cm}$ \\
\hline$\varnothing$ topo $=3 \mathrm{~m}$ & $\varnothing$ topo $=4 \mathrm{~m}$ & $\varnothing_{\text {topo }}=5 \mathrm{~m}$ \\
\hline Øintermediário $=5 \mathrm{~m}$ & Øintermediário $=5 \mathrm{~m}$ & Øintermediário $=5 \mathrm{~m}$ \\
\hline$\varnothing$ base $=30 \mathrm{~m}$ & Øbase $=36 \mathrm{~m}$ & $\varnothing$ base $=42 \mathrm{~m}$ \\
\hline $\mathrm{N}$ tubos $=13$ & $\mathrm{~N}$ tubos $=13$ & $\mathrm{~N}$ tubos $=17$ \\
\hline $\begin{array}{l}\text { Øtubos primários }= \\
323,8 \mathrm{~mm}\end{array}$ & $\begin{array}{l}\text { Øtubos primários }= \\
355,6 \mathrm{~mm}\end{array}$ & $\begin{array}{l}\text { Øtubos primários }= \\
355,6 \mathrm{~mm}\end{array}$ \\
\hline $\begin{array}{l}\text { Øtubos secundários }= \\
168,3 \mathrm{~mm}\end{array}$ & $\begin{array}{l}\text { Øtubos secundários }= \\
219,1 \mathrm{~mm}\end{array}$ & $\begin{array}{l}\text { Øtubos secundários }= \\
219,1 \mathrm{~mm}\end{array}$ \\
\hline
\end{tabular}
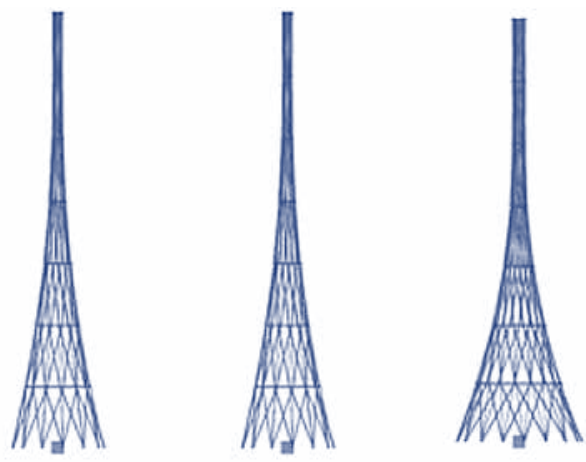

Análise MEF - 2 etapa

Nesta próxima etapa serão testadas geometrias com barras para uma espessura de $8 \mathrm{~cm}$ da casca, onde será selecionada para a análise de tensóes algumas geometrias possíveis dentro dos critérios estabelecidos.

\section{Conclusão}

O projeto performativo é colaborativo, interdisciplinar, multiprocessual e complexo. Essa abordagem de projeto exige uma mudança de cultura entre projetistas. No caso de aplicação ao desenvolvimento de uma forma estrutural, arquitetos e engenheiros devem trabalhar em um processo colaborativo, em que as informaçóes devam ter seu fluxo facilitado para todos os envolvidos na tomada de decisões. A junção de diferentes disciplinas envolvidas no projeto apresenta melhorias no desempenho da estrutura gerada, o que impacta diretamente em questôes relativas ao custo de construção. As tecnologias digitais baseadas em algoritmos propiciam uma facilidade de manipulação da forma da estrutura, o que dentro do processo de avaliação, cria métodos que permitem a racionalização do conceito através de dados numéricos. Através dos modelos gerados, podem ser avaliados os aspectos de carga, o que permite uma extrapolaçáo da forma, até se atingir os resultados desejados para uma otimização estrutural.

\section{Agradecimentos}

Os autores agradecem o apoio da CAPES para a realização da pesquisa que deu origem a este trabalho.

\section{Referências}

Burry, J., Burry, M., (2010). The New Mathematics of Architecture. Nova Iorque: Tames\& Hudson.

Pottmann, H., Asperl, A., Hofer, M., Kilian, A., (2007). Architectural Geometry. Bentley Institute Press. Exton, Pennsylvania.

Kolarevic, B., (2005). Performative Architecture beyond instrumentality. Nova Iorque: Spon Press.

Oxman, R.; Oxman, R. (Editores Convidados), (2010). The New Structuralism: design, engineering and Architectural Technologies. In: Architectural Design. Wiley, Londres.

VALLOUREC TUBOS DO BRASIL (2014). Tubos Estruturais, Seção Circular, Quadrada e Retangular. Catálogo 2014. 